\title{
A Perspective on Computational Intelligence Education
}

$\int \mathrm{o}$ oe Rothermich completed a M.Sc. in Natural Computation from the University of Birmingham, UK. He is now working in industry as a Vice President at Lincoln Vale, LLC, an alternative asset management firm.

Q. Can you give us a brief description of your education history?

A. I started with a Bachelor of Science degree in Systems Analysis at Miami University at Oxford, Ohio. The program was focused on analytical problem solving and model building with additional coverage of business and computer science topics. My Master's Degree (M.Sc.) was in Natural Computation [1] from the University of Birmingham, UK. In this course, I studied systems in nature such as evolution, complexity science, and social insects, and applied those ideas to solving problems in computation and business.

Q. Why did you decide to get a master's degree in computational intelligence?

A. I worked for several years as a consultant, first at Accenture on enterprise software and then at a smaller boutique consulting firm working on customer relationship management applications and business process design. I really enjoyed my work and the experience in development, project management and strategy for a diverse set of clients and industries. During this time I increasingly became interested in emerging technologies and quantitative topics.

Digital Object Identifier 10.1109/MCI.2009.932256
While the work I was doing was always challenging, I started to consider more intellectually stimulating work and became interested in pursuing research oriented projects. I was always interested in predictive modeling and artificial intelligence. When I started to explore the field I discovered the concept of bioinspired computing.

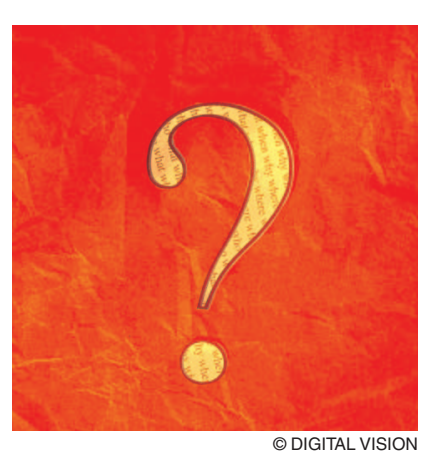

field gives you valuable perspective and can help you conceptualize specific The subject fascinated me so I talked to people working in the area. I attended a conference on evolutionary computing, where I met my eventual thesis advisor and learned about the program in natural computation that I attended. I decided that studying computational intelligence would allow me to build on my experience in industry and work on more innovative and research based projects.

Q. Do you think a Bachelor degree in computational intelligence would have been (1) beneficial and (2) necessary?

A. When I started the M.Sc. in Natural Computation, some education or experience in a technical field such as computer science was assumed. We were expected to have a background in areas such as mathematics, statistics and programming. A Bachelor's in Computational Intelligence may have been helpful, however people with diverse backgrounds contributed additional perspectives and skill sets to the program. I think gaining experience in a separate applications of what you're studying. It also helps to work with people from other backgrounds, who approach problems from different directions.

Q. Could a series of specialized workshops and seminars have compensated for an actual graduate degree?

A. A master's will allow you to go into significant depth in many areas, especially if the degree includes a thesis. Workshops and seminars will give you exposure to a field, but won't allow you to immerse yourself as deeply as you would in master's degree program. That being said, workshops and seminars can be a great start to sample the field and give you a more precise idea of what you'd like to pursue further.

Q. How did your employment path match with your education achievements?

A. My education fit very well with my eventual career path. The bachelor's in systems analysis was a great preparation for working on consulting engagements analyzing complex business processes and technology requirements. When I finished my master's, I first worked at Icosystem Corporation and then Natural Selection, Inc., both of which are research and consulting services companies that use bio-inspired computing to solve real-world problems in industries 
such as defense, commercial and biotechnology. I am currently at Lincoln Vale, LLC, an alternative asset management firm where I am working on financial models using computational intelligence.

Q. Can you offer our readers examples of real-world projects that benefited from your computational intelligence studies?

A. The majority of projects I have been directly involved with remain confidential, due to non-disclosure agreements or government sensitivity. This is common in industrial settings and makes it difficult to describe details about real-world applications. However, there are a few projects that I can discuss publicly.

For example, Natural Selection, Inc. was involved with a project sponsored by the National Science Foundation to develop software for the discovery and analysis of microRNAs in eukaryotes. MicroRNAs are small nucleic acid sequences that are expressed in certain tissues and are now understood to play a large role in cellular development and even some diseases such as cancer. Only several hundred of these microRNA genes have been experimentally verified in humans, and there is currently a great deal of interest towards the identification (and verification) of novel microRNA genes. Using computational intelligence/pattern recognition methods coupled with new "deep sequencing" methods for validation, Natural Selection, Inc. was able to identify hundreds of novel small RNA genes. Some of this content is currently being used by Invitrogen on their $\mathrm{NCode}^{\mathrm{TM}}$ Multi-Species miRNA Microarray for sale to the research community. The computational intelligence approaches were a hybridization of several different methods and were a key part of this effort.

A project that I enjoyed while working at Icosystem was work funded under DARPA to create distributed algorithms for robotic swarms. The goal was to study methodologies of creating and assessing complex swarming behaviors, particularly as applied to a building-clearing mission: a swarm of small robots enter a building, jointly create a map of their environment and search for an object of interest. Once the object is found, the swarm monitors the building for intruders and protects the object of interest. This project was carried out both in simulation and on real robots. The hardware of the robots was very limited, having only error-prone infrared sensors to detect their environment and share that information with the rest of the swarm. We created algorithms inspired by social insects so that the robots could work together to perform their task, even while limited to byte sized messages and limited sensory input. In the end we were able to program swarms that generated fairly accurate maps and were robust in that if individual robots failed, the swarm could still complete its mission.

Q. How do you think CI education can be enhanced?

A. I learn by doing and found that hands-on experience was extremely helpful to me during my master's degree. For example, one of my favorite classes was Nature-Inspired Optimization. We were assigned a well known problem, such as the traveling salesperson problem (find the shortest path through a number of cities), and each week we learned a new technique such as evolutionary algorithms, ant-colony optimization, and simulated annealing, and applied it to that same problem. Project teams presented solutions to the class on a weekly basis, creating a feedback loop that allowed us to compare algorithms and their implementations. This type of process was very effective for me and I think it can be applied in many courses.

One of the key strengths of the MSc program I attended was the level of industry involvement where students were encouraged to work with the program's industrial partners. I worked at BT in their research group (now called the Pervasive ICT Research Centre [3]) while completing my thesis on evolved distributed optimization algorithms. The people at BT were very smart and welcoming and it was a great way to learn more about the industrial applications of the field. This was one of the highlights of my educational experience and I would encourage students to seek out similar opportunities.

If you know you would like to study CI with a focus on a specific application area, it may be possible to find a program that has a strong reputation in that area and has good interdisciplinary ties between departments. There are now some programs that have built-in interdisciplinary aspects, such as a master's in computational economics [2].

Q. If you were to re-do your education path again, what would you do differently?

A. I was very happy with my path. I had the benefit of having several years of professional experience before starting my graduate degree and was able to better target my interests and career goals. Working before entering the master's program gave me the discipline to focus once I chose my direction. For someone thinking about a similar direction, I would recommend working professionally for a few years before starting a graduate degree and taking the time to thoroughly research the field, especially attending conferences and events. Most importantly, reaching out to people in the field is critical. Informational interviews, networking, and meeting new people can help a great deal when defining and targeting goals.

Q. Final words for a person considering a CI degree?

A. Computational intelligence is a broad field with many applications-invest the time to find the degree and program that best fits your interests. Talk to as many people as possible in industry and academia. Once you've started, take full advantage of the program, consider some interdisciplinary coursework, and enjoy yourself?

\section{References}

[1] X. Yao, "A research-led and industry-oriented MSc program in natural computation," IEEE Computat. Intell. Mag., vol. 1, no. 2, pp. 39-40, 2006

[2] U. Kaymak, "An MSc program in computational economics with a focus on computational intelligence," IEEE Computat. Intell. Mag., vol. 1, no. 2, pp. 41-41, 2006.

[3] BT Pervasive ICT Research Centre. Available: http:// labs.bt.com/pict/ 